\section{New syringe type of microtitre apparatus for virus and other serological tests}

J. G. ALEXANDER AND J. TEAL From the Virus Laboratory, Castle Hill Hospital, Cottingham, Yorkshire

Until now the standard micromethod for viral serological tests has been that of Takátsy (1955) or one of its modifications. The method employed in this laboratory is a miniature version of the standard macromethod (Bradstreet and Taylor, 1962), using $0.1 \mathrm{ml}$ volumes. Their method has been compared with the version to be described. Diluent, complement, antigen, and red cell suspension were added by means of a Hamilton repeating dispenser in 20 microlitre volumes, the apparatus giving 50 such volumes per fill. A Marburg syringe with a disposable yellow tip was used to add sera and to make doubling dilutions. These items ${ }^{1}$ of apparatus are obtainable in Britain and are cheaper than the apparatus described by Takátsy (1955) or its modifications.

Disposable microtitre plastic plates with U-shaped wells were used. The yellow tip on the Marburg syringe was rinsed exactly as a standard automatic syringe is rinsed, but a 'blow-out' mechanism was incorporated in the Marburg syringe.

These items of apparatus were tried out in the routine virus and other serological tests carried out in this laboratory. The antigens routinely used were influenza $\mathrm{A}, \mathrm{B}$, and $\mathrm{C}$, parainfluenza 1, psittacosis LGV, respiratory syncytial, mumps $\mathrm{S}$, mumps $\mathrm{V}$, Herpes simplex and measles viruses, adenovirus, Rickettsia burneti, Mycoplasma pneumoniae, Leptospira icterohaemorrhagiae, canicola, and biflexa.

In 93 screening tests at $1 / 10$ and 197 titrations $(1 / 10$ to $1 / 320)$ the results were shown to agree with those obtained using the macromethod. No differences of more than one dilution were observed in the titrations, and, where such differences occurred, they were reproducible and made no difference to the degree of rise in titre. There were no false positive or false negative results in the screen tests.

This microtechnique has now been adopted exclusively. It may not be quicker than the Takátsy method, but reagents are delivered as accurate volumes and not as drops, and the method is more convenient and more easily carried out by junior staff.

\section{REFERENCES}

Takátsy, G. (1955), Acta. microbiol. Acad. Sci. hung. 3, 191. Bradstreet, C. M. P., and Taylor, C. E. D. (1962). Mth. Bull. Minist. Hlth Lab. Serv., 21, 96.

${ }^{1}$ V. A. Howe \& Co. Ltd., London.

Received for publication 12 November 1968.

\section{Letters to the Editor}

Having followed with interest the correspondence concerning radioisotopic dilution techniques for vitamin $B_{12}$ assay we would like to describe a modification to one of the techniques which improves its accuracy and reproducibility.

Using the technique of Lau, Gottlieb, Wasserman, and Herbert (1965) for the assay of vitamin $B_{12}$ activity in serum, in which the intrinsic factor control is prepared using saline, we observed, as did Raven, Walker, and Barkhan (1966) and subsequently Downer and Oliver (1968), that occasionally the lower vitamin $B_{12}$ 'test' net count rates were higher than the intrinsic factor control net count rates. This anomaly will result in the theoretical calculation of a negative serum vitamin $B_{12}$ activity as well as a false lowering of vitamin $\mathbf{B}_{12}$ activities generally, since the intrinsic factor control net count rate represents the maximal count rate of isotopic labelled vitamin $B_{12}$ before dilution with 'cold' vitamin $\mathbf{B}_{12}$. Falsely low values are particularly undesirable when vitamin $B_{12}$ activities in the low normal range are reduced, somewhat haphazardly, to pathological values. At least two attempts to overcome this difficulty have been described. Raven et al (1966), and subsequently Downer and Oliver (1968), substituted the lowest vitamin $\mathbf{B}_{\mathbf{1 2}}$ 'test' net count rate in place of the intrinsic factor control net count rate in the calculation of the serum vitamin $\mathbf{B}_{\mathbf{1 2}}$ activities in any batch of assays in which this difficulty was encountered. This occasional and unpredictable substitution must produce erratic and unreproducible values. More recently, Raven et al (1967) reported experiences using a $1: 10$ dilution of a vitamin $B_{12}$-deficient serum in the preparation of the intrinsic factor control. However, we feel that this is not the complete answer, since intrinsic factor has been shown to exhibit varying binding properties of vitamin $B_{12}$ in different solutions, especially in solutions of varying concentrations of serum extracts (Rothenberg, 1968).

While attempting to find a suitable serum or protein medium for the preparation of the intrinsic factor control we accidentally observed that serum to which ascorbic acid had been added for the purpose of 'folate assay' gave consistently low vitamin $\mathrm{B}_{12}$ values by the technique of Lau et al (1965). This led to the adoption, after further investigation, of an ascorbic-acid-saturated serum as the medium used to prepare the intrinsic factor control. Using this medium we have found, in a series of over 1,500 investigations, no serum that has given a higher net count rate than the intrinsic factor control net count rate and the difficulty outlined above has therefore never been experienced. Further, we find that when this treated serum is used to prepare the intrinsic factor control it gives consistent net count rates from week to week, and any newly prepared batch of treated serum also gives net count rates that correspond accurately with those obtained from the previous intrinsic factor control.

A further important consequence of this effect of 